Ann. Génét. Sél. anim., I976, 8 (2), I53-I72.

\title{
GENES POUR LA COULEUR DE LA TOISON DE LA BREBIS CORSE
}

\author{
J.-J. LAUVERGNE et S. ADALSTEINSSON
}

Département de Génétique animale, Centre national de Recherches zootechniques, I. N. R. A., 78350 Jouy en Josas (France)

Institut de Recherches agronomiques, Keldnaholt v/Vesturlandsveg, Reykjavik (Islande)

\section{RÉSUMÉ}

La coloration pigmentaire proprement dite, c'est-à-dire le mode de répartition des deux pigments mélaniques noir et rouge dans la toison du mouton Corse serait commandée par une série de 6 allèles au locus $A$ gouti. Cinq de ces allèles: $A^{w h}$ (blancou rouge), $A^{g}$ (gris), $A^{b}$ (blaireau), $A^{w}$ (noir et feu), a (noir) ont déjà été isolés, en particulier en Islande, le sixième, que l'on a appelé oil rouge (symbole $A$ re) est, semble-t-il, particulier à la Corse.

Un variant dit rouge foncé rencontré peu fréquemment est peut-être lui aussi dû à un mutant en Agouti.

La panachure blanche du mouton Corse semble, pour l'essentiel, causée par l'action du mutant $S^{b}$ au locus de Panachure $S$. Ce mutant donne sur fond rouge la panachure irrégulière alors que, sur fond non rouge, il donne le dessin dit "points blancs " (liste en tête, extrémités des pattes et de la queue).

Ces résultats ont été obtenus par simple examen visuel de brebis et d'agneaux dans trois troupeaux localisés sur la côte orientale de l'île. Pour le locus Agouti on a raisonné par homologie avec la situation déjà observée en Islande après avoir pu montrer que le noir n'était pas induit par le mutant dominant $E^{d}$ au locus d'Extension E. Pour la panachure on a raisonné par homologie avec le comportement de $S^{b}$ récemment étudié dans des croisements entre les races Berrichonne, Solognote et Bizet.

Sur la base d'une dominance de $A^{w h}$ sur tous les allèles en $A$ gouti, d'une récessivité totale de $a$ vis-à-vis des autres allèles de la même série et d'une co-dominance des autres allèles on a estimé les fréquences des allèles de la série $A$ gouti en Corse : $A^{\text {wh }}$ (blanc ou rouge) est le plus fréquent $(0,34)$ puis viennent $a\left(\right.$ noir : o,3), et $A^{w}$ (noir et feu) : o, I5). Les fréquences de $A^{g}, A^{b}$ et $A^{\text {re }}$ sont inférieures à 0,10 . Le nombre total d'animaux impliqués dans ce calcul est de 743 .

L'hypothèse selon laquelle $S^{b}$ serait plus ou moins dominant sur fond rouge et récessif sur fond non rouge a été testée, elle est acceptable. La fréquence du gène pourrait alors être estimée à 0,67 sur toute la population.

Si l'on pense que 6 des 9 mutants de coloration décelés entre la Corse et l'Islande sont communs aux ovins de ces deux îles on doit admettre que les deux populations dérivent d'une même origine, ce qui peut aider à éclaircir l'historique du peuplement ovin ancien de l'Europe. 


\section{INTRODUCTION}

Les populations ovines de la Corse ont, selon QuiTTET (I965), suffisamment de caractères en commun pour qu'on puisse parler d'une race Corse, à vocation principalement laitière, qui, actuellement, compterait environ r Io ooo têtes (ANoNYME, I975).

La brebis Corse, jusqu'à présent, s'est maintenue pratiquement indemne de tout croisement. Elle présente une grande variété de phénotypes colorés. Ces phénotypes n'ont encore été que très sommairement décrits (cf. QuirTET, I965).

$\mathrm{Au}$ cours d'une première visite en I974 l'un d'entre nous (LAUVERGNE, I975 $a$ ) avait pu voir que cette variabilité rappelait celle analysée par ADALSTEINSSON (I970) de la brebis Islandaise. Cette remarque est à l'origine, en septembre I975, d'une courte mission en Corse au cours de laquelle les deux auteurs ont réuni les éléments de la présente étude.

\section{MATÉRIEL ET MÉTHODES}

Dans les limites d'un séjour de 5 jours dans l'île on a eu tout d'abord à régler sur place le choix de l'échantillon de troupeaux à examiner, avec tout ce que cela implique de démarches et d'organisation matérielle. Sur place encore il a fallu mettre au point une échelle phénotypique valable pour la brebis Corse, avant de recueillir les données.

L'interprétation des données a consisté, quant à elle, à établir, en premier lieu, la correspondance phénotype-génotype avant de procéder à des études de génétique de population.

\section{A. -- Le choix des troupeaux à inventorier}

Compte tenu de l'époque de l'année (en septembre les troupeaux transhumants n'ont pas encore agnelé et sont en pleine remue) il fallait prendre des troupeaux non transhumants. Pour ne pas disperser nos efforts, il fallait disposer de troupeaux relativement importants et pas trop éloignés les uns des autres afin de pouvoir travailler à partir d'une seule base d'opération. Il fallait, en outre, être sûr de trouver sur place la bonne volonté et l'assistance matérielle nécessaire au recueil des données.

Pour toutes ces raisons et sur les conseils des spécialistes de la SOMIVAC (i) notre choix s'est porté sur la zone de plus grande densité des troupeaux non transhumants, la zone médio-orientale de l'île, au niveau d'Aléria.

Les troupeaux choisis ont été :

- le troupeau du Pénitencier de Casabianda, commune d'Aléria,

- le troupeau du GAEC (groupement agricole d'exploitation en commun) de Santiolano, également dans la commune d'Aléria,

- le troupeau de la Station d'Élevage ovin d'Altiani, au pont d'Altiani, commune d'Altiani, à $\mathbf{I} 7 \mathrm{~km}$ à l'est de Corse, dans la vallée du Tavignano.

La localisation de ces points d'échantillonnage est donnée sur la carte de la figure. I.

(1) SOMIVAC. Société pour la mise en valeur agricole de la Corse, Montesoro, B. P. 226, Bastia, Corse. 


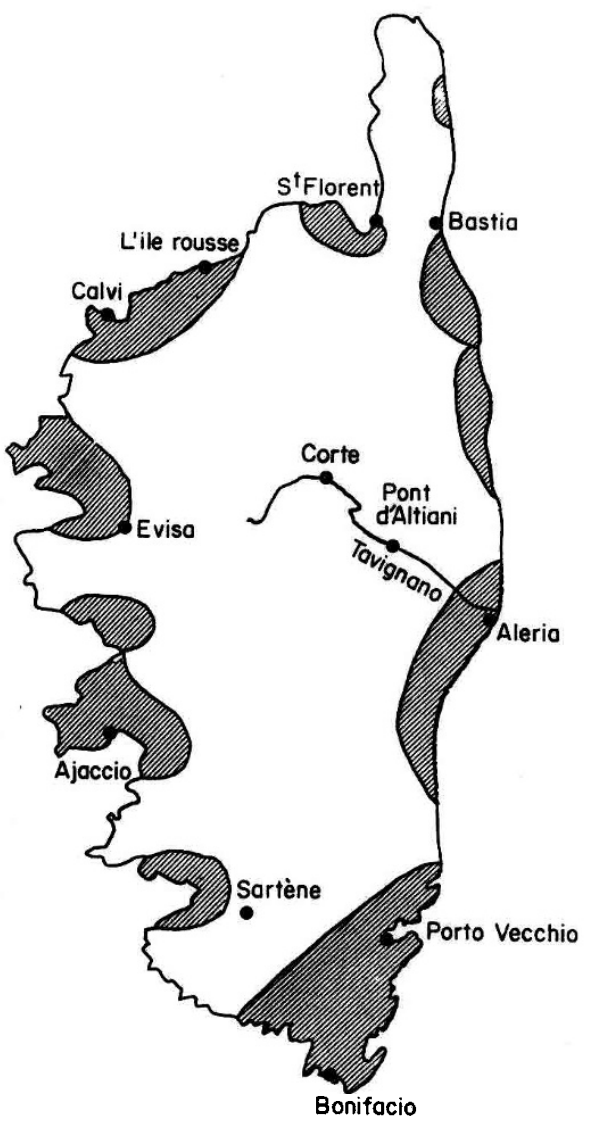

FIG. I. - Localisation des troupeaux examinés sur une carte donnant la répartition des moutons Corses pendant l'hivernage (octobre à mai)

L'agnelage débute fin août pour les troupeaux qui restent dans les zones basses, un peu plus tard pour les troupeaux qui transhument. Les laiteries, toutes situées dans les zones basses, commencent à ouvrir en octobre, elles fabriquent surtout des fromages qui seront affinés en France continentale, dans les caves de Roquefort.

Location of sampled flocks on a map giving

in Corsica the distribution of the ovine population during wintering

Lambing begins at the end of August for flocks remaining in the low areas, later for flocks that move. The dairy factories, all located in low areas, start to open in october, they make mainly cheeses to be refine in continental France, at Roquefort.

\section{B. - Les données recueillies}

A Altiani, où l'agnelage n'avait pas encore commencé, on a observé les seules brebis d'élevage (266 têtes).

A Casabianda et à Santiolano, où l'agnelage était déjà bien avancé, on aurait pu observer à la fois les brebis et leurs agneaux, mais cela n'aurait pas présenté que des avantages. Tout d'abord, dans le temps limité qui nous était imparti (une séance de 2 à 3 heures le matin, avant le départ pour le pâturage, pour chacun des élevages) on n'aurait pu voir qu'un nombre limité de familles avec, au total, moins d'individus que dans un examen limité à une catégorie (brebis ou agneaux).

Cette opération, en outre, n'aurait été possible qu'en bousculant par trop le déroulement du travail matinal des bergers. Il y avait aussi les riques d'erreurs. Pour finir, l'intérêt de con- 
TABLEAU I

Principaux phénotypes dus aux allèles au locus Agouti chez le mouton (d'après ADALSTEINSSON, 1970)

Main phenotypes due to alleles at the Agouti locus in the Sheep (from Adalsteinsson, I970)

\begin{tabular}{|c|c|c|c|c|}
\hline & \multicolumn{3}{|r|}{ Phénotypes } & \multirow{3}{*}{$\begin{array}{l}\text { Formule génique } \\
\text { en } A \text { gouti }\end{array}$} \\
\hline & \multicolumn{2}{|c|}{ Nomenclature } & \multirow[b]{2}{*}{ Description } & \\
\hline & anglaise & française & & \\
\hline 1 & white or $\tan$ & blanc ou rouge & $\begin{array}{l}\text { Varie du tout blanc au tout rouge sous } \\
\text { l'action de modificateurs. }\end{array}$ & $A^{w h} A^{w h}, A^{w h}-$ \\
\hline 2 & grey & gris & $\begin{array}{l}\text { Les poils primaires sont noirs, les sous-poils } \\
\text { sont blancs. Il y a beaucoup de variation, } \\
\text { quelquefois les sous-poils sont rouges. }\end{array}$ & $\begin{array}{l}A^{g} A^{g} \\
\text { ou } A^{g} a\end{array}$ \\
\hline 3 & $\begin{array}{c}\text { black } \\
\text { badgerface }\end{array}$ & $\begin{array}{l}\text { blaireau noir } \\
\text { ou blaireau }\end{array}$ & $\begin{array}{l}\text { Ventre noir (jarre et sous-poil) avec extension } \\
\text { du noir sur la surface interne des cuisses } \\
\text { autour de l'anus et sur la face interne de } \\
\text { la queue, sur la poitrine, la face interne } \\
\text { des pattes, le dessous du cou en bande } \\
\text { étroite et sous la mâchoire inférieure, } \\
\text { l'intérieur des oreilles est noir. Quelque- } \\
\text { fois il y a deux barres noires entre les } \\
\text { yeux. Les parties non noires sont blanc } \\
\text { sale ou rouges pour le jarre, le sous-poil } \\
\text { étant souvent noir. }\end{array}$ & $\begin{array}{l}A^{b} A^{b} \\
\text { ou } A^{b} a\end{array}$ \\
\hline 4 & $\begin{array}{l}\text { black } \\
\text { mouflon } \\
\text { ou black } \\
\text { reversed } \\
\text { badgerface }\end{array}$ & noir et feu & $\begin{array}{l}\text { Le ventre est blanc sale avec, parfois, un } \\
\text { sous-poil noir. Les zones blanches s'éten- } \\
\text { dent en arrière à l'intérieur des cuisses } \\
\text { autour de l'anus et sur la face interne de la } \\
\text { queue. La poitrine est blanche et quelque- } \\
\text { fois une bande blanche va de la poitrine à } \\
\text { sous le cou. Le dessous de la mâchoire infé- } \\
\text { rieure est blanc. Il y a une marque blanche } \\
\text { autour de l'œil. L'intérieur des oreilles est } \\
\text { également clair. Parfois le blanc sale est } \\
\text { remplacé par du rouge. La partie supé- } \\
\text { rieure du corps est principalement noire } \\
\text { avec quelquefois des poils zonés à la nais- } \\
\text { sance. Le sous-poil est noir lui aussi. }\end{array}$ & $\begin{array}{l}A^{w} A^{u} \\
\text { ou } A^{w_{a}}\end{array}$ \\
\hline 6 & $\begin{array}{c}\text { grey } \\
\text { badgerface }\end{array}$ & blaireau/gris & $\begin{array}{l}\text { Même chose que "blaireau noir " (No } 3 \text { ) sauf } \\
\text { que, dans les zones noires, le sous-poil est } \\
\text { blanc. }\end{array}$ & $A^{b} A^{g}$ \\
\hline 7 & $\begin{array}{l}\text { grey } \\
\text { mouflon }\end{array}$ & noir et feu/gris & $\begin{array}{l}\text { Même chose que " mouflon noir" (No 4) sauf } \\
\text { dans les zones noires où le sous-poil est } \\
\text { blanc. }\end{array}$ & $\begin{array}{l}A^{w} A^{g} \\
\text { ou } A^{g u}-\end{array}$ \\
\hline 9 & $\begin{array}{c}\text { black } \\
\text { mouflon } \\
\text { badgerface }\end{array}$ & $\begin{array}{c}\text { noir et feu/ } \\
\text { blaireau }\end{array}$ & $\begin{array}{l}\text { Les plages rouges des deux patrons ( } 3 \text { et } 4) \\
\text { sont conservées, au détriment des parties } \\
\text { noires. Il subsiste une raie sur le flanc, } \\
\text { quelques marques en tête. Parfois les plages } \\
\text { rouges sont blanc sale. }\end{array}$ & $A^{b} A^{w}$ \\
\hline 11 & black & noir & Tout noir, jarre et sous-poil. & $a a$ \\
\hline
\end{tabular}


naître le phénotype maternel est obéré par le fait que la paternité des agneaux ne peut être déterminée avec précision dans le système d'élevage Corse où plusieurs béliers, en général de colorations différentes, sont mélangés au troupeau.

On s'est contenté d'observer un échantillon de brebis à Casabianda (220 brebis) et un échantillon d'agneaux à Santiolano (257 agneaux).

\section{C. - La détermination de l'échelle phénotypique}

Cette échelle a été élaborée lors d'un examen préliminaire sur le troupeau de Casabianda le plus nombreux de toute la Corse. On s'est basé sur les dernières acquisitions en matière de génétique de la coloration des ovins (AdAlsteinsSON, I970 ; LAUVERGNE, 1975) en séparant la pigmentation proprement dite des dessins de panachure blanche comme des considérations, valables d'ailleurs pour tous les Mammifères, nous y autorisaient (SEARLE, I968).

\section{r. Les phénotypes pigmentés et les génotypes correspondants.}

Un phénotype pigmenté ou " patron " pigmenté est constitué par l'alternance, aussi bien à l'intérieur du poil que dans la toison elle-même, des deux pigments mélaniques noir (ou eumélanine), et rouge (ou phoeomélanine). A une extrémité de la gamme on trouve des animaux tout

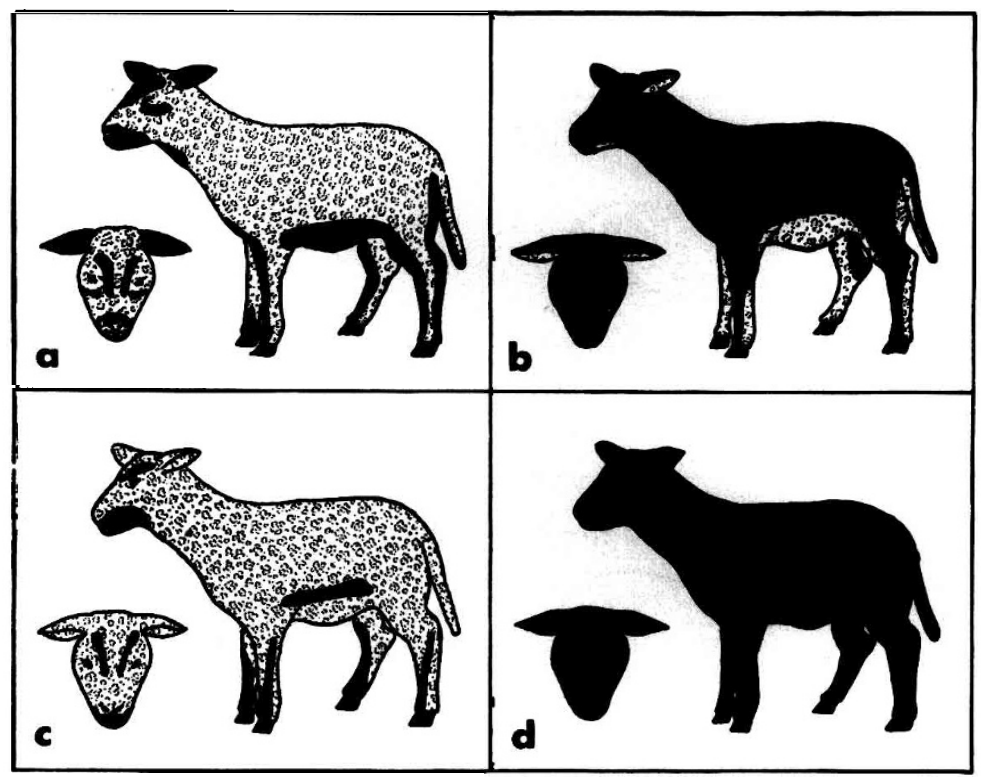

FIG. 2. - Quelques phénotypes pigmentés contrôlés par des allèles au locus Agouti chez le mouton (d'après Adalsteinsson, (1970)

a) blaireau ou blaireau noir (en gros : dos rouge ou blanc ventre noir);

b) noir et feu (en gros : dos noir ventre rouge ou blanc) ;

c) noir et feu/blaireau (combinaison des deux patrons précédents avec, en gros, maintien du noir dans les seules zones où il existe à la fois dans les deux patrons ; d) noir.

Some pigmented phenotypes controlled by alleles of locus Agouti in the sheep (from Adalsteinsson, 1970)

a) badger face (grossly white or red back, black belly);

b) black mouflon (grossly black white or red belly);

c) badger-face/mouflon (mixture of both patterns with, grossly, bearing of black in the only areas where it is present in the both patterns);

d) black.

pigmenté de mélanine noire

pigmented with black melanine

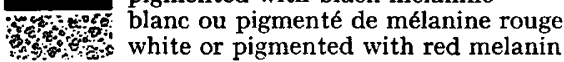




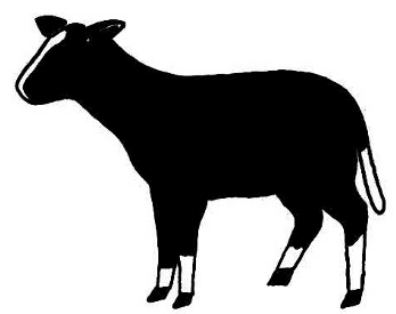

FIG. 3. - Le phénotype "points blancs". Ce phénomène est caractéristique de la race Bizet, il ne s'observe que sur fond non rouge, de préférence sur le noir ou le mouflon noir, il est dû au gène appelé $\mathrm{S}^{\mathrm{b}}$, au locus de Panachure $\mathrm{S}$

The phenotype "white points " caracterizing the Bizet breed:

This phenotype is practically only seen on non red ground : black or black mouflon. It is due to the $\mathrm{S}^{\mathrm{b}}$ gene at the Piebald locus

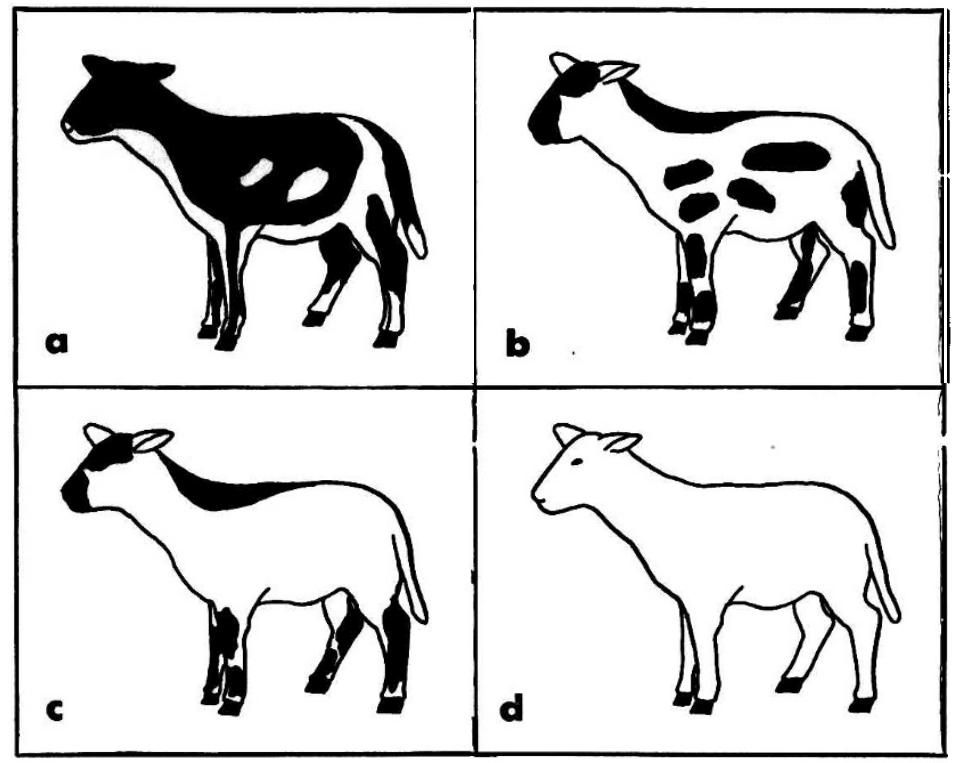

FIG. 4. - Phenotype "panachure irrégulière " tel qu'il est induit par le gène $\mathrm{S}^{\mathrm{b}}$ au locus de Panachure $\mathrm{S}$ agissant sur fond rouge.

L'intervalle de variation va de $: a$ ) rouge avec peu de blanc à $d$ ) tout blanc, en passant par $b$ ) moyennement blanc et $c$ ) beaucoup de blanc

Irregular piebald phenotype as induced by the $\mathrm{S}^{\mathrm{b}}$ gene at the Piebald locus $\mathrm{S}$ acting on tan background.

The range of variation goes from : $a$ ) tan with a few white to $d$ ) all white with b) moderately white and $c$ ) much white 
rouge et, à l'autre, des animaux tout noir. Dans l'intervalle il existe une séric d'associations pigmentaires possibles qui se répètent, avec quelques variantes, d'une espèce de Mammifères à l'autre.

En raisonnant par homologie avec la situation Islandaise on peut admettre que, chez le mouton, les patrons rouge, gris, blaireau noir, mouflon noir, blaireau gris, moufion gris et blaireau/ mouflon noir sont déterminés par les combinaisons des allèles suivants de la série $A g o u t i: A$ wh (tan), $A^{w}$ (black mouflon) $A^{\theta}$ (grey), $A^{b}$ (badger face), a (black), selon la terminologie d'ADALSTEINSSON (1974). tableau 1 .

La correspondance phénotype/génotype au locus Agouti chez le mouton est donnée dans le

La figure 2 illustre par des croquis quelques phénotypes.

En ce qui concerne maintenant le noir uniforme l'un d'entre nous (LAUvergne, I975) a définitivement démontré, semble-t-il, qu'il pouvait résulter non seulement de l'action du facteur $a$ de la série Agouti à l'état homozygote, comme nous venons de le voir, mais également, de la présence, au locus d'Extension, du mutant $E^{d}$ à la fois dominant sur l'allèle sauvage $E^{+}$et épistatique sur toutes les combinaisons alléliques au locus Agouti.

Comme le noir uniforme existe dans la race Corse il faut donc, en premier lieu, savoir quelle en est la formule génétique. Cette détermination a pu être faite à Casabianda, en même temps que l'on établissait l'échelle colorée.

\section{Les dessins blancs.}

Après Lauvergne (1975) il est possible de faire correspondre deux types de dessins blancs au même génotype, selon que celui-ci opère sur fond pigmenté rouge ou noir. Ce résultat a été obtenu après qu'ADALsTeinsson (I970) ait mis en évidence sur le mouton Islandais l'existence d'un allèle récessif de panachure à expressivité variable et irrégulière sur fond noir et pour lequel il a proposé le terme de $s$, au locus $S$, par homologie avec une situation observée chez les Rongeurs, principalement le Cobaye.

Le mutant décrit par LAUvergne (1975) en race Bizet agit sur fond rouge en donnant un dessin blanc irrégulier mais, sur fond noir, il donne un dessin limité à une liste blanche en tête et aux extrémités des pattes et de la queue : le dessin " points blancs ". La variabilité de l'expressivité sur fond rouge aurait été utilisée pour obtenir le blanc total, ce qui serait le cas pour la race Berrichonne, par exemple. Pour ce mutant Lauvergne (1975) a proposé le symbole $S^{b}\left(S^{+}\right.$ étant l'allèle normal, $b$ étant l'initiale du Bizet, la race noire à partir de laquelle la mise en évidence de l'allèle a eu lieu) pour ne pas avoir à préjuger de la dominance ; celle-ci étant variable, selon le fond pigmenté de la toison et l'accumulation des modificateurs polygéniques. Le symbole $S^{b}$ a été utilisé dans la présente étude.

Dans la population Corse il sera donc tout indiqué de rechercher d'abord les marques blanches des deux types évoqués ci-avant. P'our le point blanc de type Bizet, cela ne prête pas à confusion (cf. fig. 3).

Pour la panachure irrégulière et le tout blanc on a imaginé une échelle en quatre degrés inspirée de l'échelle donnée par LAUVERGNE (I969) (fig. $4 a, b, c, d$ ).

$\mathrm{Au}$ cas où l'on retrouverait le comportement décrit plus haut la correspondance phénotype/génotype serait alors celle donnée dans le tableau 2.

\section{TABLEAU 2}

Différents phénotypes panachés dus au mutant $S^{b}$ en interaction avec les génotypes en Agouti

Various piebald phenotypes induced by $S^{b}$ interacting with Agouti genotypes

\begin{tabular}{|c|c|c|}
\hline \multirow{2}{*}{ Génotypes } & \multicolumn{2}{|c|}{ Phénotypes } \\
\hline & $A^{w h}$ présent & $A^{w h}$ absent \\
\hline $\begin{array}{l}S^{+} S^{+} \\
S^{+} S^{b} \\
S^{b} S^{b}\end{array}$ & $\begin{array}{l}\text { rouge uniforme } \\
\text { pie rouge } \\
\text { pie rouge ou tout blanc }\end{array}$ & $\begin{array}{l}\text { non blanc uniforme } \\
\text { non blanc uniforme } \\
\text { points blancs }\end{array}$ \\
\hline
\end{tabular}




\section{D. - Les études de génétique des populations}

On a préféré ce terme plus général à un titre qui ferait allusion à la simple estimation des fréquences géniques. C'est qu'en effet nos données sont telles que l'on doit faire une étude groupée dans laquelle on estime les fréquences géniques tout en testant tel comportement des gènes (de dominance et de pénétrance) et telle ou telle situation de l'équilibre génique. Faute de données suffisantes ce cas se rencontre souvent en génétique des populations mais il n'était pas inutile de le rappeler.

Ainsi a-t-on postulé que les conditions de HARDY-Weinberg étaient réalisées : équilibre des fréquences géniques atteint, choix au hasard des reproducteurs. La méthode utilisée a alors été celle du maximum de vraisemblance. Quant aux situations de dominance, elles devront être examinées dans le cas de chaque locus, après que l'on ait fait la correspondance phénotype/ génotype. Des développements spéciaux seront éventuellement nécessaires, ils seront donnés à ce moment-là.

\section{RÉSULTATS}

\section{4. - Les phénotypes de la brebis Corse et les génotypes correspondants}

\section{r. Phénotypes et génotypes pigmentés.}

a) La formule des moutons noirs.

Au Pénitencier de Casabianda, on utilise depuis quelques années un certain nombre de béliers Sardes, tout blancs. Les $\mathrm{F}_{\mathbf{1}}$ sont facilement reconnaissables à leur taille et à leur port de tête. Tous ceux que nous avons pu observer étaient de phénotype tout blanc ou panaché rouge, même ceux dont la mère était noire ou pie noir. Cela prouve que le noir que l'on rencontre quelquefois en Corse est dû à la présence de l'allèle $a$ (noir) au locus $A$ gout $i$ et non de l'allèle dominant et épistatique $E^{a}$. Au passage on peut penser qu'à l'instar de 1a race Berrichonne blanche étudiée par LAUVERGNE (r975) la race ovine Sarde, elle aussi toute blanche, est de formule $E^{+} E^{+} A^{w h} A^{w h} S^{b} S^{b}$, tenant son phénotype blanc de $1^{\prime}$ interaction de $A^{w h}$ avec $S^{b}$ (cf. tabl. 2).

\section{b) Les nouveaux phénotypes et génotypes colorés.}

Outre les phénotypes énumérés dans le tableau I on a observé en Corse 4 phénotypes pigmentés nouveaux. Leur description avec une tentative de nomenclature, est donnée dans le tableau 3. A la figure 5 on donne le croquis correspondant à deux de ces phénotypes.

Faute de résultats de ségrégation l'interprétation factorielle de ces phénotypes ne peut être définitive. Cependant, si l'on laisse de côté pour le moment le rouge foncé (sooty) qui était vraiment peu fréquent on peut postuler que le phénotype noir avec la tache rouge autour de l'œil est un dessin induit par un nouvel allèle au locus Agouti. Cet allèle serait dominant par rapport à $a$ (noir) et récessif vis-à-vis de $A^{w h}$ (rouge) et de $A^{b}$ (blaireau noir) tout en étant co-dominant avec $A^{w}$ (noir et feu) et $A^{g}$ (gris) (cf. tabl. 4 pour la nomenclature allélique).

Les raisons que nous avons pour faire apparaître ce nouvel allèle seront analysées en détail dans la discussion. On n'a mentionné ici cette interprétation que parce qu'elle était indispensable pour la suite de l'exposé des résultats. 
TABLEAU 3

Nouveaux phénotypes pigmentés ovins observés en Corse New sheep pigmented phenotypes observed in Corsica

\begin{tabular}{|c|c|c|c|c|}
\hline \multirow[t]{2}{*}{ No } & \multicolumn{2}{|c|}{$\begin{array}{c}\text { Nomenclature } \\
\text { proposée }\end{array}$} & \multirow{2}{*}{ Description } & \multirow{2}{*}{$\begin{array}{l}\text { Possible } \\
\text { formule } \\
\text { en } A \text { gout }\end{array}$} \\
\hline & anglaise & française & & \\
\hline 1 & $\begin{array}{l}\text { black } \\
\text { red eye }\end{array}$ & $\begin{array}{l}\text { noir } \\
\text { à œil } \\
\text { rouge }\end{array}$ & $\begin{array}{l}\text { Tout le corps est noir sauf une } \\
\text { tache rouge autour de l'œil. }\end{array}$ & $\begin{array}{l}A^{r e} A^{r e} \\
A^{r e} a\end{array}$ \\
\hline 2 & $\begin{array}{l}\text { grey } \\
\text { red eye }\end{array}$ & $\begin{array}{l}\text { gris/ } \\
\text { oil rouge }\end{array}$ & $\begin{array}{l}\text { Même chose que noir à ceil rouge } \\
n^{\circ} 5 \text { avec sous-poil blanc dans les } \\
\text { zones noires. }\end{array}$ & $A^{r e} A^{r e}$ \\
\hline 3 & $\begin{array}{c}\text { black } \\
\text { mouflon- } \\
\text { red eye }\end{array}$ & $\begin{array}{l}\text { noir } \\
\text { et feu/ } \\
\text { œil rouge }\end{array}$ & $\begin{array}{l}\text { Même chose que noir et feu avec } \\
\text { extension plus marquée d'une } \\
\text { zone rouge autour de l'œil. }\end{array}$ & $A^{w} A^{r e}$ \\
\hline 4 & sooty & rouge foncé & $\begin{array}{l}\text { Tout le corps est couvert de poils } \\
\text { d'un rouge intense dont la pointe } \\
\text { est noire sur une certaine lon- } \\
\text { gueur, le phénotype est assez } \\
\text { variable. }\end{array}$ & \\
\hline
\end{tabular}

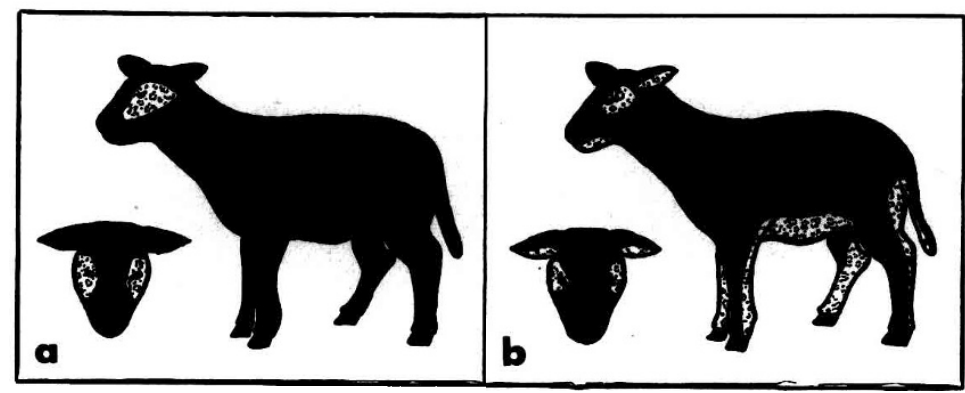

Fig. 5. - Les deux phénotypes pigmentés nouveaux observés en Corse

a) noir à œil rouge

b) noir et feu-œil rouge

The two new pigmented phenotypes observed in Corsica

a) black red eye

b) black mouflon-red eye 


\section{Les phénotypes et génotypes de panachure blanche.}

En matière de panachure blanche on n'a observé pratiquement en Corse que des phénotypes correspondant à l'action du mutant $S^{b}$ évoqué ci-avant. Les quelques exceptions qui consistent en des animaux pie noir seront analysées au chapitre discussion.

\section{Un bilan des mutants déjà décelable en Corse.}

Dans le tableau 4 est donnée la liste des mutants décelés dans l'échantillon des trois troupeaux que nous avons inventoriés dans la zone d'Aléria. A titre de comparaisons on donne également la liste des mutants détectés en Islande, d'après ADALSTEINSSON (I974).

\section{TABLEAU 4}

Les allèles responsables de la coloration de la toison du mouton en Corse et en Islande

Alleles inducing fleece coloration in Corsican and Icelandic sheep

\begin{tabular}{|c|c|c|c|c|c|c|}
\hline \multicolumn{3}{|c|}{ Loci } & \multicolumn{2}{|c|}{ Symboles des allèles décelés } & \multicolumn{2}{|c|}{ Nomenclature des mutants } \\
\hline \multirow[b]{2}{*}{ Symbole } & \multicolumn{2}{|c|}{ Nomenclature } & \multirow[b]{2}{*}{ en Corse $\left({ }^{1}\right)$} & \multirow[b]{2}{*}{ en Islande $\left(^{\mathbf{2}}\right)$} & \multirow[b]{2}{*}{ anglaise } & \multirow[b]{2}{*}{ française } \\
\hline & anglaise & française & & & & \\
\hline$A$ & A gouti & A gouti & $\begin{array}{l}\mathrm{A}^{w i z} \\
A^{w} \\
A^{g} \\
A^{b} \\
A^{r e} \\
a\end{array}$ & $\begin{array}{l}A^{w h} \\
A^{g w} \\
A^{w} \\
A^{g} \\
A^{b} \\
a\end{array}$ & $\begin{array}{l}\text { white or tan } \\
\text { grey mouflon } \\
\text { mouflon } \\
\text { grey } \\
\text { badger face } \\
\text { red eye } \\
\text { self colour } \\
\text { (black or brown) }\end{array}$ & $\begin{array}{l}\text { rouge ou blans } \\
\text { noir et feu } \\
\text { gris } \\
\text { blaireau } \\
\text { ail rouge } \\
\text { noir }\end{array}$ \\
\hline$B$ & Brown & Brun & $B^{+}$ & $\begin{array}{l}B \\
b\end{array}$ & $\begin{array}{l}\text { wild type black } \\
\text { brown }\end{array}$ & $\begin{array}{l}\text { sauvage noir } \\
\text { brun }\end{array}$ \\
\hline$E$ & Extension & Extension & $E^{+}$ & $E$ & wild type & sauvage \\
\hline$S$ & Piebald & $\begin{array}{c}\text { Panachure } \\
S\end{array}$ & $\begin{array}{l}S^{+} \\
S^{b}\end{array}$ & $\begin{array}{l}S \\
s\end{array}$ & $\begin{array}{l}\text { wild type } \\
\text { piebald }\end{array}$ & $\begin{array}{l}\text { sauvage } \\
\text { bizet }\end{array}$ \\
\hline
\end{tabular}

(1) Symbolisation qui s'écarte de celle d'Adalsteinsson (1974) pour tenir compte de l'allèle sauvage marqué + quand il est connu.

(2) Terminologie d'Adalsteinsson (197'́).

\section{B. - Génétique des populations}

\section{I. - Présentation des données.}

Conformément à la division : actions pigmentaires et dessins blancs on a imaginé un tableau à double entrée avec, horizontalement, les types pigmentaires et, verticalement, les dessins blancs. Le tableau 5, donne les comptages à Casabianda, Altiani et Santiolano. 
GÈNES DE COULEUR DE LA BREBIS “ CORSE 》

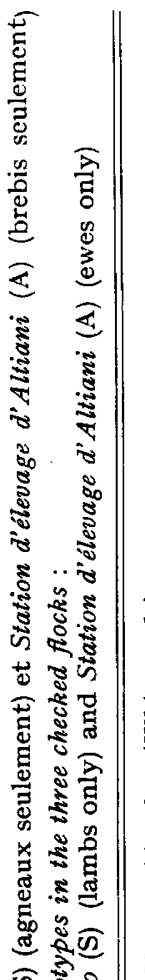

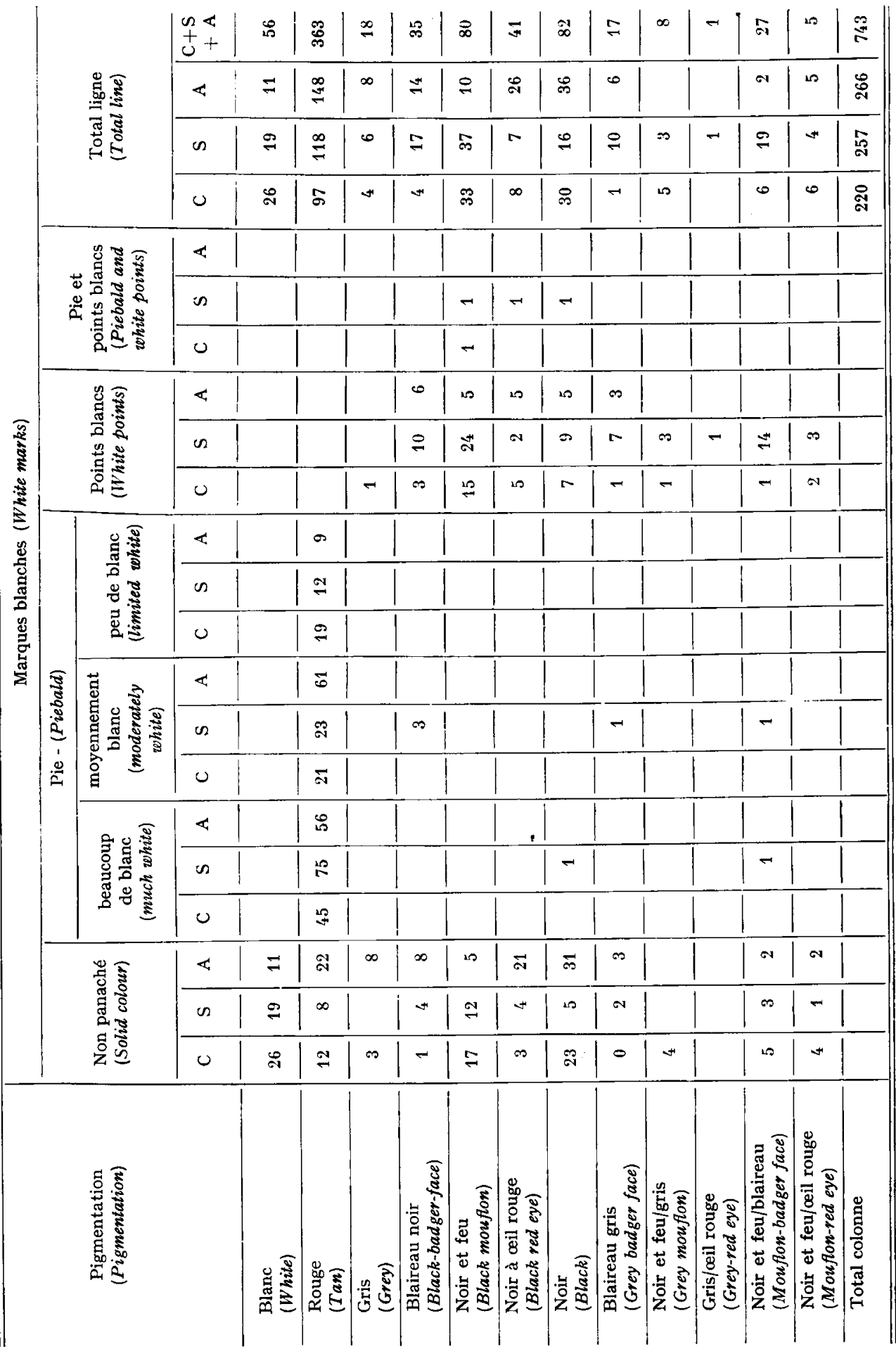


2. Étude de la situation au locus Agouti A.

On a seulement retenu 9 classes phénotypiques pour l'estimation des 6 allèles décelés : blanc ou rouge, gris, blaireau noir, noir et feu, noir à œil rouge, noir, blaireau/gris, noir et feu/gris et blaireau/noir et feu.

Les classes gris à œil rouge et noir et feu à œil rouge ont été respectivement combinées avec gris et noir et feu. Il était en effet difficile de distinguer le patron œil rouge sur fond gris ou noir et feu, surtout chez les animaux adultes.

Ainsi l'équation d'estimation correspond à une situation sextiallélique dans laquelle un gène domine tous les autres, trois allèles sont codominants entre eux $\left(A^{g}, A^{b}\right.$ et $\left.A^{w}\right)$ et dominants sur les deux derniers allèles $\left(A^{r e}\right.$ et $\left.a\right)$ à l'exception de $A^{w}$, codominant avec $\mathrm{A}^{r e}$, l'avant-dernier allèle qui, lui-même, est dominant sur le dernier $(a)$.

L'estimation a été faite dans chaque troupeau séparément dans l'hypothèse de HARDY-WEINBERG (panmixie, avec équilibre génique) comme il a été dit précédemment.

Les fréquences géniques sont données dans le tableau 6 dans lequel on a également fait figurer des estimations de fréquences faites sur le troupeau Islandais, à titre de comparaison.

\section{TABLEAU 6}

Estimées des fréquences géniques au locus Agouti

Gene frequency estimates at the A locus

\begin{tabular}{|c|c|c|c|c|c|c|c|c|c|c|}
\hline \multirow{3}{*}{ Allèles } & \multicolumn{6}{|c|}{ Troupeaux corses } & \multirow{2}{*}{\multicolumn{2}{|c|}{$\begin{array}{c}\text { Corse } \\
\text { troup. groupés }\end{array}$}} & \multirow{2}{*}{\multicolumn{2}{|c|}{ Islande }} \\
\hline & \multicolumn{2}{|c|}{ Casabianda } & \multicolumn{2}{|c|}{ Altiani } & \multicolumn{2}{|c|}{ Santiolano } & & & & \\
\hline & fréq. & $\sigma^{\prime}$ & fréq. & $\sigma$ & fréq. & $\sigma$ & fréq. & $\sigma$ & fréq. ( $\left.{ }^{1}\right)$ & fréq. $\left({ }^{2}\right)$ \\
\hline$A^{w h}$ & 0,3379 & 0,0253 & 0,3658 & 0,0237 & 0,3167 & 0,0228 & $0,3{ }_{4} 02$ & 0,0138 & 0,6732 & 0,5900 \\
\hline$A^{g}$ & 0,0351 & 0,0109 & 0,0425 & 0,0111 & 0,0589 & 0,0128 & 0,0460 & 0,0068 & 0,0640 & 0,0901 \\
\hline$A^{b}$ & 0,0386 & 0,0115 & 0,0680 & 0,0140 & 0,1410 & 0,0195 & 0,0842 & 0,0091 & 0,0073 & 0,014 k \\
\hline$A^{w} \ldots$ & 0,1950 & 0,0250 & 0,0527 & 0,0124 & 0,2122 & 0,0235 & 0,1473 & 0,0119 & 0,0311 & 0,0342 \\
\hline$A^{r e} \ldots \ldots \ldots \ldots$ & 0,0439 & 0,0159 & 0,1121 & 0,0208 & 0,0450 & $0,019 / 4$ & 0,0701 & 0,0110 & & \\
\hline$a \ldots \ldots \ldots \ldots$ & 0,3495 & 0,0306 & 0,3589 & 0,0282 & 0,2262 & 0,0278 & 0,3122 & 0,0167 & 0,2193 & 0,2713 \\
\hline Nbre d'animaux.... & & & 2 & 66 & 2 & & & & 547 & 17118 \\
\hline
\end{tabular}

(1) Basée sur les comptages de croisements entre mâles non blancs avec des brebis blanches ou rouges Adalsteinsson (1970).

(2) Estimée à partir de coloration observée publiée précédemment (Adalsteinsson, 1970).

Le tableau 7 donne les effectifs de chaque classe tels qu'on peut les calculer à partir des estimations des fréquences géniques par le maximum de vraisemblance, avec les différences entre valeurs observées et valeurs calculées. 


\section{TABIEAU 7}

Fréquences calculées (C) dans chaque classe de couleur et différences entre fréquences observées $(\mathrm{O})$ et calculées $(\mathrm{O}-\mathrm{C})$

Expected frequencies (C) in each colour class and differences between observed (O) and expected frequencies $(\mathrm{O}-\mathrm{C})$

\begin{tabular}{|c|c|c|c|c|c|c|c|c|c|}
\hline \multicolumn{2}{|c|}{ Classes phénotypiques colorées } & \multicolumn{6}{|c|}{ Troupeaux } & \multirow{2}{*}{\multicolumn{2}{|c|}{$\begin{array}{l}\text { Troupeaux } \\
\text { groupes }\end{array}$}} \\
\hline \multirow{2}{*}{$\begin{array}{l}\text { Nomenclature } \\
\text { française }\end{array}$} & \multirow{2}{*}{$\begin{array}{l}\text { Nomenclature } \\
\text { anglaise }\end{array}$} & \multicolumn{2}{|c|}{ Casabianda } & \multicolumn{2}{|c|}{ Altiani } & \multicolumn{2}{|c|}{ Santiolano } & & \\
\hline & & $\mathrm{C}$ & $\mathrm{O}-\mathrm{C}$ & $\mathrm{C}$ & $\mathrm{O}-\mathrm{C}$ & C & $\mathrm{O}-\mathrm{C}$ & $\mathrm{C}$ & $\mathrm{O}-\mathrm{C}$ \\
\hline Blanc ou rouge & White or tan & 123 & 0 & 159 & 0 & 137 & 0 & 419 & 0 \\
\hline Gris & Grey & 5 & -1 & 8 & 0 & 8 & -2 & 21 & -3 \\
\hline Blaireau noir & Black badgerface & 7 & -3 & 18 & -4 & 25 & -8 & 50 & -15 \\
\hline Noir et feu & Black mouflon & 39 & -6 & 11 & -1 & 36 & 1 & 86 & -6 \\
\hline Noir à œil rouge & Black red eye & 7 & 1 & 25 & 1 & 6 & 1 & 38 & 3 \\
\hline Noir & Black & 27 & 3 & 34 & 2 & 13 & 3 & 74 & 8 \\
\hline Blaireau/gris & Grey badgerface & 1 & ) & 2 & 4 & t & 6 & 7 & 10 \\
\hline Noir et feu/gris & Grey mouflon & 3 & 2 & 1 & -1 & 7 & -4 & 11 & -3 \\
\hline Gris à œil rouge & Grey red eye & 1 & -1 & 3 & -3 & 1 & 0 & $\check{j}$ & -4 \\
\hline Blaireau/noir et feu & Badgerface mouflon & 3 & 3 & 2 & 0 & 15 & 4 & 20 & 7 \\
\hline Noir et feu à oil rouge & Mouflon red eye & 4 & 2 & 3 & 2 & $\check{5}$ & -1 & 12 & 3 \\
\hline \multicolumn{2}{|c|}{ Total $\ldots \ldots \ldots \ldots \ldots \ldots \ldots \ldots$} & 220 & 0 & 266 & 0 & 257 & 0) & 743 & 0 \\
\hline
\end{tabular}

\section{La situation au locus de Panachure $\mathrm{S}$.}

On a vu plus haut (tabl. 2) que la situation de dominance de l'allèle $S^{b}$ sur fond rouge s'inversait sur fond noir, où $S^{b}$ devenait récessif. Les données du tableau 5 , offrent la possibilité, tout en estimant la fréquence génique, de tester 1'hypothèse évoquée à l'instant : $S^{b}$ serait complètement récessif chez les animaux ne possédant pas $A^{w h}$ alors qu'il serait dominant avec une expressivité variable chez les moutons $A^{w h}$ : hétérozygotes pie rouge et homozygotes blancs ou pie rouge.

Pour l'estimation par le maximum de vraisemblance de $q$ la fréquence de $s$ on peut utiliser le procédé suivant :

$$
e^{\mathbf{L}} \propto[q(2-q)]^{n_{1}}(\mathrm{I}-q)^{2 n_{2}}\left(\mathrm{I}-q^{2}\right)^{n_{3}}\left(q^{2}\right)^{n_{4}}
$$

$\left.\mathrm{L} \propto\left(n_{1}+2 n_{4}\right) \log q+\left(2 n_{2}+n_{3}\right) \log (\mathrm{I}-q)\right)$

$$
+n_{1} \log (2-q)+n_{3} \log (I+q)
$$

En dérivant on obtient :

et

$$
\mathrm{S}=\frac{\mathrm{dL}}{\mathrm{d} q}=\frac{n_{1}+2 n_{4}}{q}-\frac{2 n_{2}+n_{3}}{\mathrm{I}-q}-\frac{n_{1}}{2-q}+\frac{n_{3}}{\mathrm{I}+q}
$$

$$
\frac{\mathrm{d}^{2} \mathrm{~L}}{\mathrm{~d} q^{2}}=-\frac{n_{1}+2 n_{4}}{q^{2}}-\frac{2 n_{2}+n_{3}}{(\mathrm{I}-q)^{2}}-\frac{n_{1}}{(2-q)^{2}}-\frac{n_{3}}{(\mathrm{I}+q)^{2}}
$$

(1) $\propto$ signifie directement proportionnel à. 
L'espérance mathématique négative de la dérivée seconde devient alors :

et

$$
-\mathrm{E}\left(\frac{\mathrm{d}^{2} \mathrm{~L}}{\mathrm{~d} q^{2}}\right)=\mathrm{N}\left(\frac{2+q}{q}+\frac{3-q}{\mathrm{I}-q}+\frac{q}{2-q}+\frac{\mathrm{I}-q}{\mathrm{I}+q}\right)
$$

$$
\operatorname{var}(q)=\frac{\mathrm{I}}{-\mathrm{E}\left(\frac{\mathbf{d}^{2} \mathrm{~L}}{\mathrm{~d} q^{2}}\right)}
$$

On extrait $q$ de l'équation de la dérivée première en faisant $S=0$. Comme il n'y a pas de solution algébrique simple, on doit envisager une itération de la forme :

$$
q_{1}=q_{0}+\mathrm{S}_{0} \operatorname{var}\left(q_{0}\right)
$$

et la poursuivre jusqu'à avoir $q$ avec 4 décimales.

\section{TABLEAU 8}

Nombres observés dans les différentes classes génotypiques de panachure blanche, estimation de $q$ (fréquence de $S^{b}$ ) par le maximum de vraisemblance et text $\gamma^{2}$ de l'hypothèse de HARDY et WEINBERG

Observed numbers in different genotypic classes for piebaldness, estimation of $q$ (frequency of $S^{b}$ ) by the maximum of likelihood and test of HARDY WEINBERG hypothesis

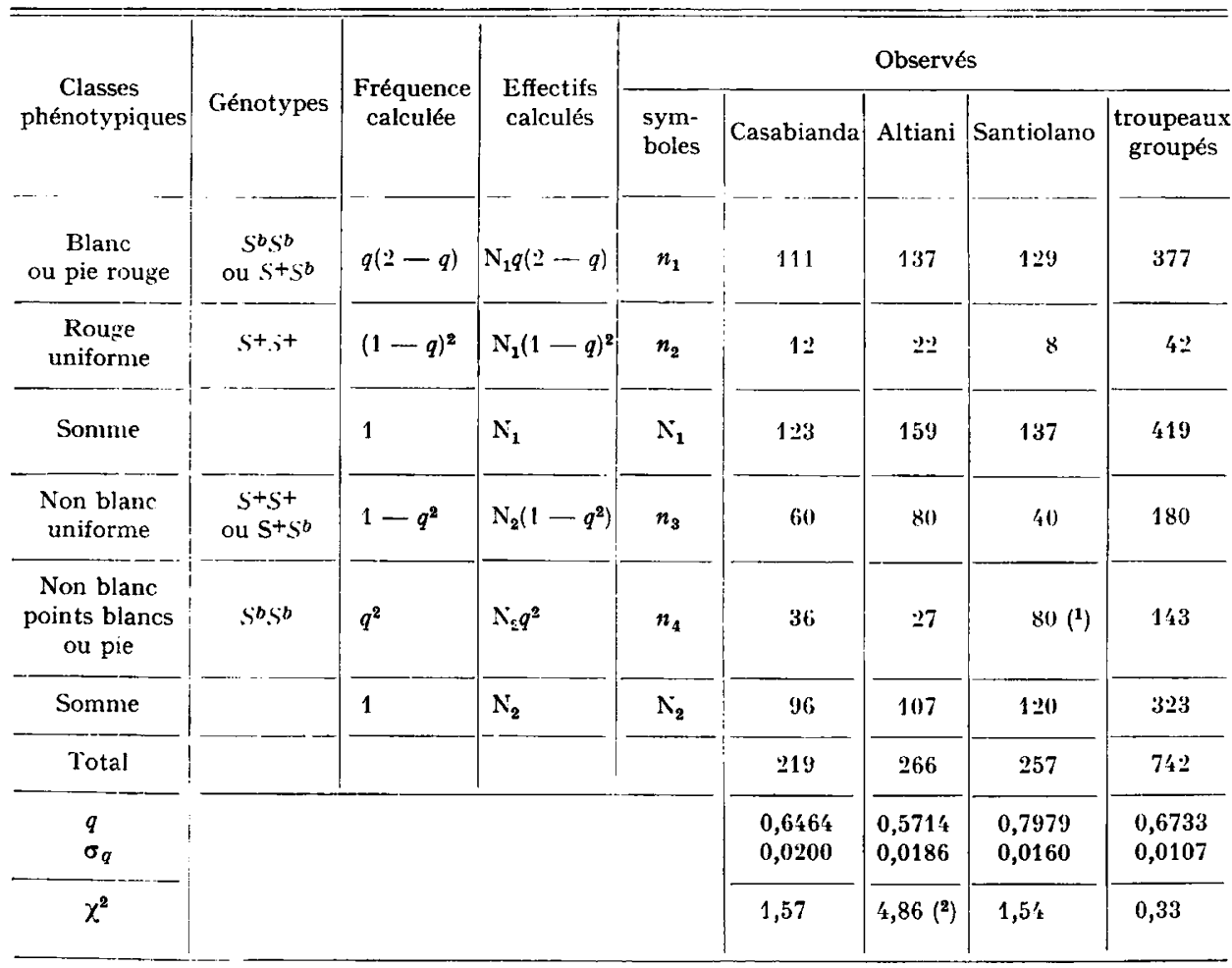

(1) Les animaux panachés sans marques en tête portant $A^{b}$, au nombre de 6 , et un agneau pie noir sont inclus dans ce total.

( $\left.{ }^{2}\right) 0,05>\mathrm{P}>0,01$. 
Dans le tableau 8 la valeur de $q$ est obtenue à l'intérieur de chaque troupeau à partir de deux groupes de données, chacun avec un degré de liberté, d'un côté pour les animaux portant $A^{w h}$, de l'autre pour ceux qui ne portent pas $A^{w h}$.

L'estimation de $q$ nécessite un degré de liberté. Le degré de liberté restant peut alors être utilisé pour tester l'ajustement entre valeurs observées et calculées dans chaque groupe de données.

Les valeurs calculées dans chaque classe pour chaque troupeau et troupeaux regroupés sont comparées aux valeurs observées par le test du $\chi^{2}$.

\section{DISCUSSION}

\section{A. - Les gènes décelés}

\section{La série Agouti.}

Sachant, comme nous l'avons plus haut, que le noir uniforme n'est pas dû à 1'allèle dominant $E^{d}$ en $E$ et raisonnant par homologie avec la situation en Islande on peut,-tout d'abord, dénombrer en Corse 5 allèles en $A$ gouti :

$$
A^{w h}, A^{g}, A^{w}, A^{b} \text { et } a .
$$

Dans les tableaux I et 3 pour les phénotypes, 4 pour les allèles on remarquera que la correspondance en français du terme " mouflon " adopté par ADALSTEINSSON (I970 et I974) en l'occurrence ici ( black mouflon » en égard à la couleur de l'eumélanine est " noir et feu ". On a ainsi voulu marquer qu'il était possible que ce patron aussi bien en race Corse qu'en race Islandaise ne soit pas le patron sauvage comme le terme "mouflon" repris d'ailleurs de BERGE (I958) le laisserait entendre mais correspondrait plutôt à l'action du mutant " noir et feu " (black and tan), $A^{t}$ au locus Agouti, très répandu chez les Mammifères. Comme, toutefois, le présent article n'est pas et ne peut pas être consacré à une étude détaillée de la série allélique en Agouti nous ne pousserons pas plus loin la discussion et continuerons en conservant pour Agouti la notation allélique d'AdaLsteinsson.

La nouveauté en Corse est $A^{\text {re }}$ qui semble pouvoir être rattaché à la série Agouti pour les raisons suivantes : I) le phénotype qu'il détermine a un "air de famille " avec les phénotypes induits par les allèles en Agouti, qui mélangent des plages noires à des plages rouges avec souvent des dessins sur la face, spécialement $A^{b}$ (blaireau) et $A^{w}$ (noir et feu). En outre, il se comporterait assez bien d'une manière co-dominante avec les autres allèles de la série pour donner les phénotypes intermédiaires décrits dans le tableau 3 et la figure 5 . Bien sûr des tests d'allélisme avec les autres allèles de la série seraient les bienvenus.

Il est plus difficile de se prononcer sur le déterminisme du " rouge foncé " (sooty). On peut penser qu'il s'agit d'un autre allèle de la série $A$ gouti proche de $A^{w h}$ qui, lui, semble induire un rouge assez délavé. Il se pourrait également que cette intensification résulte de l'action d'un facteur situé à un autre locus. La rareté des cas de "rouge foncé " trouvés fait que ces hypothèses n'ont pu être miess à l'épreuve. 


\section{La panachure blanche.}

Il n'y a guère de doute sur la présence de $S^{b}$ à une fréquence assez élevée de race Corse. Toutefois on relève un certain nombre d'exceptions au comportement que nous prêtons à ce gène sur fond noir : 4 animaux noirs portent à la fois la panachure irrégulière et les points blancs.

En outre, en supposant que les parties rouges du corps des animaux blaireau sont affectées de la même manière que leurs homologues chez les animaux $\left[A^{\text {wh }}\right]$ et les parties noires des noir et feu de la même façon que celles des noir uniforme on ne devrait pas observer de blaireau, blaireau/gris, blaireau noir ou noir et feu avec la panachure irrégulière or on en observe 7 en tout (tabl. 5).

On peut arguer qu'il s'agit d'un comportement atypique de $S^{b}$, bien que la coexistence des dessins chez un même animal rende cette hypothèse douteuse et laisserait plutôt à penser qu'un autre allèle de panachure à un autre locus pourrait être en cause.

Des éléments de vérification de cette hypothèse biallélique n'ont pu être rassemblés sur le terrain car la question n'a surgi qu'après coup. De toute façon l'examen de la panachure est rendu difficile chez les adultes où la laine pousse blanc sur le corps, masquant les éventuelles taches pigmentées. La détection pourrait être plus facile sur les agneaux avec, par exemple, photo systématique de tous les animaux panachés.

\section{Les limites dues au mode de recueil des données et à l'échantillonnage.}

On vient de voir, aussi bien à propos de la pigmentation proprement dite (avec le phénotype œil rouge) qu'à propos de la panachure, qu'il est possible de laisser passer un mutant pour peu qu'il soit rare et qu'il sorte du cadre qu'il faut bien se donner au départ.

Comme, en outre, (cf. fig. I) on n'a échantillonné que sur l'une des huit principales zones d'hivernage, cela veut dire qu'un certain nombre de variants ont pu nous échapper.

4. La comparaison avec la race Islandaise et avec quelques autres populations européennes.

Si l'on s'en tient aux seuls gènes mutés, c'est-à-dire excluant l'allèle sauvage quand il est connu, on peut voir (tabl. 4) que, sur 9 mutants de coloration décelés au total, 6 sont communs aux deux îles [ $S^{b}$ et les cinq allèles en $A$ gouti évoqués plus haut) un esṫ propre à la Corse $\left(A^{r e}\right)$ et 2 à $1^{\prime}$ Islande $\left(A^{g w}\right.$ et $\left.b\right)$.

Même s'il est possible d'envisager que certaines mutations peuvent être récurrentes cette explication ne peut valoir pour tous les allèles semblables trouvés.

On en est donc conduit à admettre que les races Islandaise et Corse ont eu, à une certaine époque, des ancêtres communs constituant déjà, si l'on en juge par le nombre des mutants de coloration accumulés, une population domestique assez évoluée.

D'après les données historiques on sait que le mouton Islandais dérive de souches Scandinaves dont les premières importations remontent à mille ans environ. Certaines variations colorées en $A$ gouti $\left(A^{w h}, A^{g}, A^{w}, A^{b}, a\right)$ se retrouvent d'ailleurs de nos jours encore chez le Vieux mouton norvégien (Spaelsau) si l'on examine les 
données de BERGE (I958, I974) et également chez le mouton de Gotland en Suède. Dans les îles au Nord de 1'Écosse, RYDER et al. (I974) ont aussi apparemment retrouvé une partie de ces allèles. Il s'agit sans doute du même courant migratoire scandinave que celui qui a peuplé 1'Islande. La nouveauté est qu'il faut maintenant considérer qu'un courant fort semblable, mais sans doute plus ancien, a peuplé la Corse. Cela laisse à penser que les bases continentales de ces mouvements d'animaux (bases qui restent à localiser mais qui sont sans doute bien au Sud de la Scandinavie) ont été jadis occupées par une population ovine bigarrée. Cette population a, par la suite, disparu (absorption ou remplacement?) de ces zones pour ne subsister que dans le nord de 1'Europe et en Corse.

İ y a là une donnée qui peut intéresser les historiens de la domestication.

\section{Une variabilité déjà perçue par les éleveurs corses.}

Jusqu'à présent les auteurs, on l'a vu, n'ont pas analysé les variations colorées de la brebis Corse, ni phénotypiquement ni, et encore moins, génotypiquement. Cela est très compréhensible : à cause sans doute de l'étrangeté des patrons, la plupart pratiquement inconnus dans les races en vogue sur le continent et aussi de la confusion qui régnait jusqu'à une date récente en matière de génétique de la coloration du mouton. Cependant, pour les éleveurs et les bergers cette variabilité est plus familière et la langue corse offre toute une terminologie. Il faudrait, pour la démêler l'aide d'un linguiste mais, déjà, on a pu voir que certains patrons en Agouti étaient bien perçus comme une entité. Une étude précise révélerait, avec sans doute certaines variantes, une terminologie semblable mais en une langue dérivée du latin cette fois, à celle qui a été analysée par ADALsTEINsson (I970) pour quelques langues nordiques (le norvégien, le suédois, l'islandais, le shetlandais) et 1'anglais.

\section{B. - Génétique des populations}

\section{I. $A$ u locus Agouti.}

Le tableau 6 montre que $A^{w h}$ et $a$ ont les fréquences les plus élevées.

Le fait le plus notable du tableau 7 est l'excès de blaireau gris et le défaut de blaireau noir dans les 3 troupeaux. Cet écart peut provenir des causes suivantes:

- un effet de la sélection : les blaireaux gris étant préférés aux blaireaux noirs, mais cela ne vaudrait que pour les 2 échantillons d'animaux adultes ;

- la présence d'un gène modificateur pour une canitie plus ou moins précoce transformant seulement la couleur noir du ventre en gris. Un tel effet, comme il n'était pas prévu, aurait fort bien pu ne pas être décelé sur les autres phénotypes;

- l'existence d'un allèle en Agouti qui combinerait les comportements de $A^{g}$ et $A^{b}$. Un tel allèle existe chez le mouton Islandais : le facteur $A^{g} w$ d'ADALSTEINSSON (I974);

- des erreurs de classification : les animaux blaireau étant faussement classés blaireau/gris.

La dernière cause avancée est sans doute la plus probable car le ventre des moutons Corses étant poilu, la distinction entre ventre noir et ventre gris s'avère difficile.

On peut voir, également dans le tableau 7 , que les animaux noir et feu/gris sont 
moins nombreux que prévu. Cela voudrait dire que $A^{g w}$ envisagé ci-dessus est soit très rare, soit absent, du matériel que nous avons examiné en Corse. C'est la raison pour laquelle nous n'avons pas mentionné ce facteur dans la liste des allèles en Agouti de Corse.

Le nouvel allèle $A^{r e}$, quant à lui, se place assez bien dans la perspective ébauchée au tableau 6. Les effectifs observés pour les noirs à œil rouge et les noir et feu/ œil rouge s'ajustent eux aussi aux valeurs attendues. Cependant qu'un seul gris/ œil rouge a été observé, pour 5 attendus. Cet animal était un jeune agneau. On a pu manquer de vigilance pour déceler ce phénotype chez les brebis, ou bien est-il plus difficilement visible chez l'adulte.

\section{Au locus $S$.}

Comme le montre le tableau 8 il y a un bon accord entre les valeurs observées et les valeurs calculées à Casabianda et Santiolano alors qu'un écart significatif s'observe à Altiani. Cela voudrait dire soit que le comportement de $S^{b}$ n'est pas le même à Altiani, soit que l'équilibre n'a pas encore été atteint dans ce dernier troupeau. Les données indiquent que la fréquence de $S^{b}$ est plus haute parmi les brebis ne portant pas $A^{w h}$ que parmi les porteuses de $A^{w h}$. Cela s'expliquerait assez bien si les animaux blancs ou rouges avaient une provenance différente de celle des animaux non blancs ou rouges. Le troupeau d'Altiani étant une sorte de synthèse entre plusieurs troupeaux locaux, cela n'est pas impossible.

\section{Les raisons du maintien de la variabilité colorée en Corse.}

La plupart du temps, lors de la formation des races modernes d'animaux domestiques, un standard coloré unique est adopté.

Cette tendance a pu même être très ancienne chez le mouton où la technologie de la teinture exige des laines blanches, c'est-à-dire totalement dépigmentées.

Ainsi les grands troupeaux qui, dès le début de la Renaissance, ont alimenté 1'industrie drapière en Europe occidentale étaient-ils déjà composés, pour l'essentiel, d'animaux blancs, de même que les troupeaux à laine fine qui supplantèrent les races européennes locales avant d'avoir le développement que l'on sait dans l'hémisphère sud.

Néanmoins les laines colorées (en noir principalement mais aussi en gris et en brun) n'ont pas cessé d'avoir des adeptes surtout - mais pas seulement - pour le tricotage.

Par ailleurs les mutants colorés peuvent avoir un certain intérêt en pelleterie. C'est le cas bien connu, pour l'Astrakan. C'est aussi le cas pour le mouton de Gotland gris qui est en vogue en Suède depuis quelques années (RYDER, I970).

Pour ces usages cependant le nombre de variants colorés conservés est en général limité. En outre, ces raisons ne valent guère pour la Corse où la laine n'intéresse plus beaucoup les gens depuis déjà longtemps et où l'utilisation des dépouilles en pelleterie a été et reste limitée.

Les vraies raisons pourraient découler de l'isolement dû à l'insularité, isolement_renforcé par le caractère montagneux de l'île, avec une intervention de type moderne qui peut paraître paradoxale.

Avec la dépopulation constante de l'île depuis bientôt un siècle (RENUCCr, I974) l'élevage ovin n'aurait pu, en effet, se maintenir jusqu'à nous avec les effectifs que 
nous lui connaissons si les industriels de Roquefort n'avaient implanté leurs usines qui expédient sur le Continent. Toutefois et jusqu'à l'aménagement récent de la plaine orientale - qui a d'ailleurs surtout été fait pour la viticulture - cet élevage ovin ne pouvait être qu'un élevage transhumant, de type extensif. Or, les possibilités d'améliorer la productivité d'un tel élevage restent, même actuellement, limitées : il se maintient tel qu'il est ou disparâit. Donc, même si le modernisme a pu atteindre le stade de traitement et de commercialisation des laits, les bergers corses, qui pouvaient subsister grâce à la stabilisation de leurs revenus, n'avaient aucune raison de modifier leurs méthodes traditionnelles d'élevage qui, outre le maintien de la variabilité de la coloration, présentent, au dire des ethnologues nombre de caractères fort archaïsants (RAvis-GroRDANI, I975).

\section{CONCLUSION}

Une telle variabilité génétique visible non encore décrite dans une race domestique française est, certes, une aubaine mais cela ne doit pas masquer le caractère encore incomplet de la présente étude, limitée à trois troupeaux, dans une seule petite zone, sans analyse des descendances.

Outre différents ajustements, il reste à faire une étude de génétique des populations sur les migrations internes des reproducteurs et son influence sur les fréquences géniques dont on essaiera de savoir quel degré d'équilibre elles ont atteint dans l'île.

Il reste aussi à exploiter l'apport que la ressemblance entre le mouton Corse et le Vieux mouton Scandinave peut avoir pour éclairer l'histoire du peuplement de l'Europe par le mouton.

Reçu pour publication en avril 1976.

\section{REMERCIEMENTS}

MM. Cerutti et Salvat de la SOMIVAC à Bastia nous ont aidés de leurs conseils dans le choix des troupeaux à échantillonner.

Sur place nous avons disposé de la coopération efficace de MM. MoRETTI responsable élevage au Pénitencier de Casabianda, FERnandez de la Station d'Élevage d'Altiani et Vincent Carlott et ses collaborateurs, du GAEC de Santiolano.

Le manuscrit a été relu par M. Ryder de l'ABRO et P. MÉrat du Département de Génétique animale de l'I.N.R.A.

\section{SUMMARY}

\section{FLEECE COLOR GENES OF THE CORSICAN EWE}

The pigmentary coloration in sheep that are characterised by patterns in the distribution of tan vs. black pigment, have been studied in the Corsican sheep. The colour patterns found in this breed were found to be determined by a series of 6 alleles at the Agouti locus. Five of these alleles have been isolated elsewhere, particularly in Iceland. They are: $A^{\text {wh }}$ (white or tan), $A^{\circ}(g r e y)$, 
$A^{b}$ (badgerface), $A^{w}$ (black mouflon) and $a$ (black). The sixth proposed allele, $A^{r e}$, producing a red patch around the eye in black, black mouflon and grey sheep, has so far only been found in Corsica.

A colour variant called "sooty" is possibly also caused by a mutant at the Agouti locus.

Broken colour among the Corsican sheep seems to be caused by a mutant gene, $S^{b}$, at the Spotting locus, $S$. This mutant allele produces tan piebald in tan sheep, but white pattern (white blaze or head spot, white socks and white tip of tail) in non-tan sheep.

The above conclusions have been reached by analysing data obtained by visual inspection of three sheep flocks on the eastern coast of the island.

As regards the $A$-locus, use has been made of the analogy with the already analysed situation in Iceland after showing that the black is not caused by the dominant mutant at the Extension $E$. locus. Comparaison with the recent studies on the breeds Berrichon, Sologne and Bizet has formed the basis for the conclusions with respect to the $S$-locus.

The following estimates of allele frequencies at the A-locus have been obtained, assuming $A^{w h}$ to be the top dominant, $a$ being bottom recessive and the other alleles intermediate and co-dominant : $A^{w h}(\tan )$ with the highest frequency $(0.34), a($ black $)$ next $(0,3 \mathrm{I})$ and $A^{w}$ (black mouflon) the third (0.I5), while $A^{g}, A^{b}$ and $A^{\text {re }}$ all had frequencies below o.Io. The calculations were based on altogether 743 sheep.

The hypothesis that $S^{b}$ is dominant on tan background and recessive on black background has been tested, and was found to be acceptable. The frequency of $S^{b}$ was found to be 0.67 .

By assuming that 6 of the 9 mutant colour genes found in Iceland and Corsica are the same mutations, it follows that the sheep populations on the two islands are of common ancestry, which, in turn, could be utilised in tracing the history of sheep domestication in ancient Europe.

\section{RÉFÉRENCES BIBLIOGRAPHIQUES}

Adalsteinsson S., r97o. Colour inheritance in Icelandic Sheep and relation between coulour, fertility and fertilization. J.agr. res. Icel., 2, 3-r 35 .

Adalsteinsson S., I974. Colour inheritance in farm animals and its application in selection. Ist World Congr. Genet. appl. anim. Prod., 1, 29-36.

Anonyme, 1975. L'élevage ovin Corse en 1974. Ministère de l'Agriculture Service central des enquêtes et études statistiques et Direction Départementale de l'Agriculture de la Corse. Ronéoté, pp. 8.

Berge S., 1958. Farger hos den gamle norske sauen. Meld. Norg. Landbrukshgsk., 37 (6), pp. Ig.

BERGE S., 1974. Sheep colour genetics. Z. Tierzucht ZüchtBiol., 90, 297-321.

LaUvergne J.-J., r975 a. Situation ethnique comparée de la Corse et de la Sardaigne en I974: bovins, ovins, caprins, porcins et équidés. In ONUAA (FAO) et PNUE, Étude pilote sur la conservation des ressources génétiques animales, FAO, Rome.

Lauvergne J. J., I975 $b$. Génétique de la couleur de la toison des races ovines françaises Solognote, Bizet et Berrichonne. Ann. Génét. Sél. anim., 7, 263-276.

Quittet E., 1965. Races ovines françaises. La Maison rustique, $2^{\mathrm{e}}$ éd., 46-47.

Ravis-Giordani G., 1975. Le troupeau errant. L'utilisation de l'espace par les bergers du Niolu (Corse). In Ethnologie et histoire, Éditions sociales, Paris, 285-30r.

Renucci Janine 1974. Corse traditionnelle et Corse nouvelle. Audin, Lyon, ro6-r3r.

Ryder M. L., 1970. Sheep in Scandinavia. Span, 13 (I), I-3.

Ryder M. L., LAND R. B., I974. Coat colour inheritance in Soay, Orkney and Shetland sheep. J. Zool. Lond., 178, $477-485$.

SEARLE, 1968. Comparative genetics of coat Colour in Mammals. Logos Press, London. 